\title{
Drug repurposing and therapeutic anti-microRNA predictions for inhibition of oxidized low-density lipoprotein-induced vascular smooth muscle cell-associated diseases
}

\author{
Shun-Tsung Chen ${ }^{* * *}$, Chien-Hung Huang ${ }^{\dagger \dagger \dagger}$, Victor C. Kok ${ }^{*+,+1}$, \\ Chi-Ying F. Huang ${ }^{\S}, \S$, Jin-Shuei Ciou* ${ }^{*}$,, Jeffrey J. P. Tsai*,|l|, \\ Nilubon Kurubanjerdjit ${ }^{\uparrow, * * *}$ and Ka-Lok $\mathrm{Ng}^{*, \|, \dagger \dagger \dagger}$ \\ *Department of Bioinformatics and Medical Engineering \\ Asia University, Taichung, Taiwan 41354 \\ ${ }^{\dagger}$ Department of Computer Science and Information Engineering \\ National Formosa University, Yun-Lin, Taiwan 63205 \\ Division of Medical Oncology \\ Kuang Tien General Hospital Cancer Center \\ Taichung, Taiwan 43303 \\ $\S$ Institute of Biopharmaceutical Sciences \\ National Yang-Ming University, Taipei, Taiwan 112 \\ "School of Information Technology \\ Mae Fah Luang University, Chiang Rai, Thailand 57100 \\ "Department of Medical Research \\ China Medical University Hospital \\ China Medical University, Taichung, Taiwan 40402 \\ **stc100218003@gmail.com \\ †chhuang@nfu.edu.tw \\ +victorkok@asia.edu.tw \\ $\S \S$ cyhuang5@ym.edu.tw

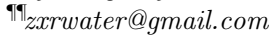 \\ IIIjjptsai@gmail.com \\ ***sendtoopal@gmail.com \\ ${ }^{\dagger \dagger}$ ppiddi@gmail.com,klng@asia.edu.tw
}

Received 30 September 2016

Revised 11 December 2016

Accepted 11 December 2016

Published 2 February 2017

Drug repurposing is a new method for disease treatments, which accelerates the identification of new uses for existing drugs with minimal side effects for patients. MicroRNA-based therapeutics are a class of drugs that have been used in gene therapy following the FDA's approval of the first

$\dagger \dagger$ Corresponding author.

This is an Open Access article published by World Scientific Publishing Company. It is distributed under the terms of the Creative Commons Attribution 4.0 (CC-BY) License. Further distribution of this work is permitted, provided the original work is properly cited. 


\begin{abstract}
anti-sense therapy. This study examines the effects of oxLDL on vascular smooth muscle cells (VSMCs) and identifies potential drugs and antimiRs for treating VSMC-associated diseases. The Connectivity Map (cMap) database is utilized to identify potential new uses of existing drugs. The success of the identifications was supported by MTT assay, clonogenic assay and clinical trial data. Specifically, 37 drugs, some of which are undergoing clinical trials, were identified. Three of the identified drugs exhibit IC50 activities. Among the 37 drugs' targets, three differentially expressed genes (DEGs) are identified as drug targets by using both the DrugBank and the NCBI PubChem Compound databases. Also, one DEG, DNMT1, which is regulated by 17 miRNAs, where these miRNAs are potential targets for developing antimiRbased miRNA therapy, is found.
\end{abstract}

Keywords: Cardiovascular disease; vascular smooth muscle cell; time-course microarray; oxidized low-density lipoprotein; differentially expressed gene; drug repurposing; microRNA; antimiR; Gene Ontology enrichment analysis.

\title{
1. Introduction
}

Cardiovascular disease (CVD) is the most important cause of mortality worldwide. CVD includes any disease that affects the heart or circulatory vessels, including atherosclerosis (ATs), hypertension, coronary artery disease, myocardial infarction and peripheral vascular diseases. This disease originates in vascular lesions (such as abnormal vasomotor tone), and such lesions often have similar causes and are associated with similar disease processes. ${ }^{1}$ According to the WHO report, CVD affects tens of millions of people each year. Therefore, a consensus concerning improving the diagnosis, treatment and prevention of CVD must be urgently established. ${ }^{2}$

The vessel wall is the integrative organ that comprises cells of three types, which are endothelial cells, vascular smooth muscle cells (VSMCs) and fibroblasts. It may self-modulate in response to various stimuli. Vascular abnormalities lead to phenotypic changes in the VSMCs, which constitute most of the walls of the blood vessel and have an important role in the pathogenesis of CVDs. Since CVDs are a very broad range of diseases, VSMC-associated diseases are used to specify diseases that are caused by phenotypic changes in the following discussion.

When the arterial vessel is subjected to risk factors (such as an oxidized lowdensity lipoprotein (oxLDL) or mechanical stress), which may initiate signaling pathways that are usually mediated by growth factors, triggering differential gene expression, and thereby inducing the dedifferentiation and phenotypic transition of VSMCs, the VSMC phenotype changes from a contractile state to a synthetic state and may promote the growth and migration of VSMC. Such a transition between different phenotypes is referred to as "phenotypic transition". It is a major cause of ATs,${ }^{3}$ restenosis,${ }^{4}$ hypertension, ${ }^{5}$ abnormal cell growth and apoptosis in VSMC. ${ }^{6,7}$

ATs that is caused by VSMC proliferation is similar to cancer formation, in which tissue mass grows as a result of inflammation and uncontrolled cell proliferation. Several studies have suggested that the pathogenesis of both ATs and cancer is caused by malfunction of the same molecular pathways. ${ }^{8-10}$ Pathways that are common to ATs and cancer (such as PI3K/Akt) can mediate several functional and morphological alterations of VSMCs after they are activated, developing vascular diseases, ${ }^{11}$ and 
affecting the growth, apoptosis and regulation of the cycles of cells of various types, inducing cancer progression. ${ }^{12}$ The MAPK pathway, which is involved in VSMC proliferation, hypertrophy and migration, and is critical to the pathogenesis of vascular diseases, ${ }^{13}$ has effects that are similar to the PI3K/Akt in the development of cancer. ${ }^{14}$

Based on the above observations, we hypothesize that some of the Food and Drug Administration (FDA)-approved cancer drugs may be repurposed for treating the VSMC-associated diseases. The repurposing of drugs may minimize side effects and accelerate drug development for cancer therapy. ${ }^{15}$ This work aims to identify drug molecules that may reverse VSMCs proliferation if the drugs reverse disease-induced signatures. The "signatures" means the "gene expression profiles" herein.

To identify such drugs, both up- and down-regulated differentially expressed genes (DEGs) were used to query the Connectivity Map ${ }^{16}$ (cMap) database. cMap, a web-based resource, is a robust tool that addresses queries by using five cell lines (MCF7, ssMCF7, PC3, HL60 and SKMEL). As stated by Lamb et al., ${ }^{17}$ "The breast cancer epithelial cell line MCF7 has been extensively used as a reference in laboratories around the world. A subset of perturbagens was also profiled in the prostate cancer epithelial cell line PC3 and the non-epithelial cell lines HL60 (leukemia) and SKMEL (melanoma). This variety of cell types provides a chance to estimate the extent to which results are relied upon the experimental conditions for proper interpretation." Even though meeting all conditions is impossible, the results obtained using various cell lines are acceptable to most of the researchers.

The dysregulation of microRNAs (miRNAs) expression causes many diseases, including cancer. MiRNAs may modulate gene expression and protein synthesis by negative regulation, by base pairing with the 3 '-UTR of target mRNAs, which regulate many specific biological processes (BPs) (such as cell development, differentiation, signaling, metabolism and stress responses). Several specific miRNAs - miR$21,{ }^{18} \mathrm{miR}-143 / 145^{19}$ and miR-221/222 ${ }^{20}$ — have been identified as actively participating in the regulation of VSMC. In particular, miR-145 has been experimentally verified as having an anti-proliferative effect when over-expressed..$^{21}$ The above results reveal that miRNAs have a critical role in the regulation of the VSMC phenotype transition and cause VSMC-associated diseases. Disease-associated miRNAs are a class of targets for developing miRNA-based therapies in which anti-sense oligonucleotides (anti-microRNAs antimiRs) are used to inhibit miRNA expression. ${ }^{20,22}$

MiRNA-based therapeutics are another class of drugs that are used in gene therapy. With the recent FDA approval of the first anti-sense therapy for treating hyperlipidemia, miRNA-based therapy has demonstrated its clinical effectiveness. ${ }^{23}$ Recent progress in the pharmacological inhibition of disease-associated miRNAs offers great promise in developing novel miRNA-based therapeutics. For example, in three in vivo studies by Marquart et al. ${ }^{24}$ Najafi-Shoushtari et al. ${ }^{25}$ and Rayner et al. ${ }^{26}$ antimiR was used to probe the functions of miR-33 in cholesterol homeostasis. Those investigations demonstrated the inhibition of miR-33 by antimiR-33, which elevates circulating high-density cholesterol levels, increases reverse cholesterol transport and reduces ATs. Even though the development of 


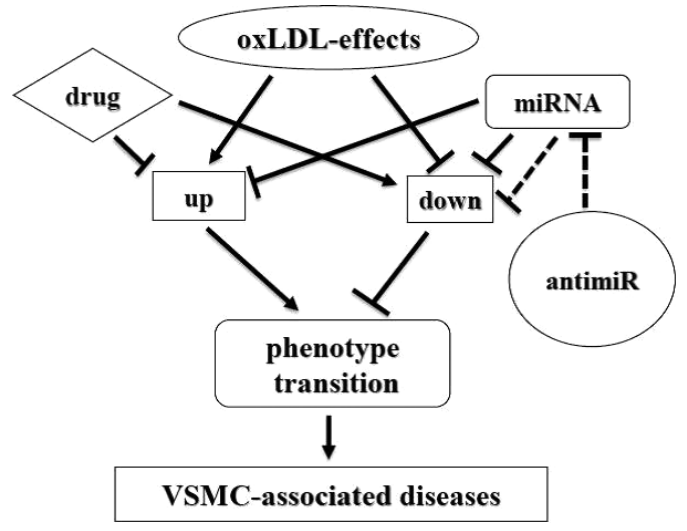

Fig. 1. The concept for the treatment of oxLDL-induced VSMC-associated diseases. 'Up' and 'down' denote the up- and down-regulated DEGs, respectively. The dotted line denotes the interactive relationships among antimiR, miRNA and down-regulated DEGs. The arrows and the blunts denote activation and suppression, respectively.

antimiR-based miRNA therapeutics has had positive effects, it also has some side effects. The current design and use of antimiRs may have undesirable effects when antimiR-mediated is used to suppress miRNA expression, such as the down-regulation of RNA species other than the intended miRNA.

Figure 1 presents the concept for the treatment of VSMC-associated diseases. OxLDL induces both up- and down-regulated DEGs, which in turn trigger a VSMC phenotypic transition. Drugs could inhibit up-regulated DEGs or activate downregulated DEGs, preventing VSMC phenotypic transition.

Aberrant over-expressed miRNA could silence specific target genes and thereby promote the VSMC phenotypic transition. AntimiR can be synthesized to silence the over-expressed miRNA, restore the down-regulated DEGs expression (dotted line in Fig. 1) to normal levels, stop the VSMC phenotypic transition, and prevent VSMCassociated diseases. On the other hand, miRNA can be introduced into the patient to target up-regulated DEGs, hence, inhibits the expression of up-regulated DEGs; restoring them to a normal level, and thereby suppress the VSMC phenotypic transition. This work examined the antimiR-mediated miRNA inhibition case.

The field of miRNA pharmacogenomics concerns the synergistic effects of miRNAs and the efficacy of drugs, which have potential clinical implications for personalized medicine. ${ }^{27}$ For example, Shi et al. ${ }^{28}$ reported a dual-drug delivery system that comprises miR-34a and paclitaxel has the potential for improving cancer therapy. Such a synergistic effect is not considered herein.

\section{Methods}

This study develops a strategy for identifying oxLDL-induced DEGs, potential drugs and therapeutic antimiRs based on microarray data. Figure 2 presents the workflow. 


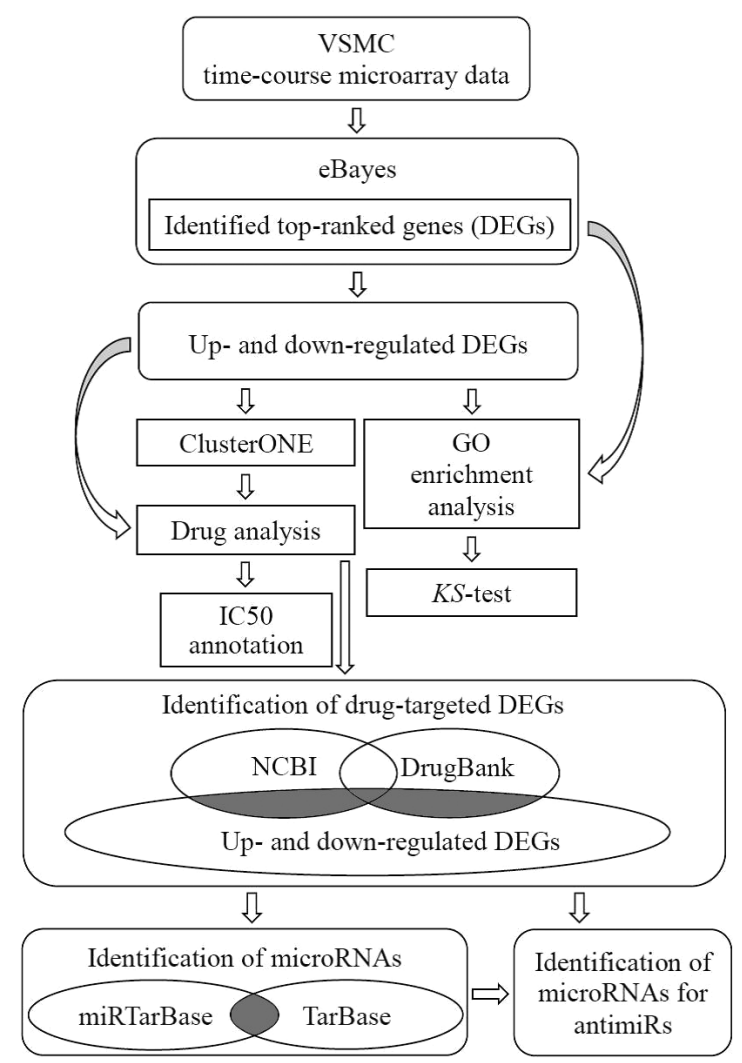

Fig. 2. Workflow of this study.

\subsection{Datasets}

To examine how oxLDL contributes to the response of VSMCs and to study its underlying signal transduction pathways, the microarray experiment GSE13139 from the Gene Expression Omnibus ${ }^{29}$ was used. This experiment measured changes in gene expression that are activated by the binding of oxLDL to the Lectin-like ox $L D L$ receptor $(L O X-1)$ of endothelial cells at five time points of $0,2,6,12$ and $24 \mathrm{~h}$. Each time point measurement was repeated twice to examine the temporal patterns of the gene expression in response to oxLDL. Each sample comprised three replicates that were prepared from HAECT cells.

\subsection{Identification of DEGs and clustering analysis}

In this work, Bioconductor ${ }^{30}$ was utilized for identifying DEGs. First, the Robust Multi-Array Average (RMA) is used to normalize gene expression. Then, a model matrix is created (using the function, model.matrix) with rows and columns that denote the replicates (with information obtained at the five time points) and the time 
points. Next, we seek a linear model that describes each probe/gene using the lmFit function that is provided in the limma package. Finally, the function, cont.matrix, is utilized to construct a contrast matrix, which enables a pairwise comparison of time points among the three replicates (using the function contrast.fit).

Many statistical methods exist for identifying DEGs, of which the significance analysis of microarrays (SAM), ${ }^{31,32}$ the empirical Bayes analysis of microarrays (EBAM), ${ }^{33,34}$ and the empirical Bayes statistics (eBayes) ${ }^{35}$ are commonly used. In a previous study, ${ }^{36}$ we found that eBayes, SAM and EBAM identified cancer genes with similar accuracy of around $20 \%$. Therefore, eBayes is used herein to identify top-ranked DEGs with assumed adjusted $p$-values, $p_{\text {eBayes }}$, of less than $5 \%$. The eBayes algorithm overcomes the possibility of identifying genes with small foldchanges that may be statistically significant.

The eBayes algorithm ${ }^{37}$ computes moderated $t$-statistics, moderated $F$-statistics, and log-odds of differential expression by the empirical Bayes shrinkage of the standard errors towards a common value. The moderated $t$-statistic is defined by

$$
\tilde{t}_{p q}=\frac{\hat{B}_{p q}}{\tilde{s}_{p} \sqrt{C_{p q}}},
$$

where $p$ and $q$ are the $p$ th gene and the $q$ th time, respectively and the contrast estimator $\hat{B}_{p q}$ denotes the difference between two classes. $\tilde{S}_{p}$ is the estimated shrinkage of the standard deviation of the $p$ th gene expression level, and $C_{p q}$ is the $p$ th diagonal element of the covariance matrix.

In this study, we focus on genes that are consecutively up- or consecutively downregulated in four time intervals ( 0 versus $2 \mathrm{~h}, 2$ versus $6 \mathrm{~h}, 6$ versus $12 \mathrm{~h}$ and 12 versus $24 \mathrm{~h}$ ), because these genes are unambiguously up- or down-regulated. We define these as the DEGs. We hypothesize that a drug molecule in cMap may potentially reverse the disease signature if the drug-induced gene expression profile is significantly negatively correlated with the disease-induced signature. Accordingly, given two sets of disease-induced DEGs, i.e. both up- and down-regulated DEGs, anti-correlated drug-induced signatures in cMap were sought.

Furthermore, we made use of both up- and down-regulated clusters to identify the drugs. It is because genes belong to the same cluster are typically involved in similar BPs. A graph clustering algorithm called "clustering with overlapping neighborhood expansion" (ClusterONE) ${ }^{38}$ is used to generate both up- and downregulated clusters. ClusterONE deals with weighted graphs and yield overlapping clusters for predicting potentially protein complexes in protein-protein interaction (PPI) networks. The parameters provided by ClusterONE are grouped into two modes - i.e. basic (minimum size, minimum density, edge weights) and advanced (node penalty, haircut threshold, merging method, similarity, overlap threshold, seeding method). Default values are used in the present study, i.e. basic (3, auto, unweighted) and advanced (2, 0, single-pass, match coefficient, 0.8, from unused nodes). 
The procedure for identifying clusters is described as follows: first, given both up- and down-regulated DEGs and the PPI information from BioGRID, ${ }^{39}$ the up-regulated (down-regulated) PPI network is extracted as the maximal subnetwork from BioGRID consisting entirely of up-regulated (down-regulated) genes. Second, ClusterOne was run on the up-regulated (down-regulated) PPI network to generate up-regulated (down-regulated) clusters. Only clusters with $p$-values,

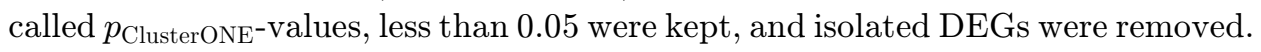
Third, both up- and down-regulated clusters were combined in pairs to query cMap.

\subsection{Gene ontology enrichment analysis}

DAVID $^{40}$ is a web-based resource, which accepts a gene list and performs Gene Ontology (GO) $\mathrm{BP}^{41}$ and Kyoto Encyclopedia of Genes and Genomes (KEGG) pathway $^{42}$ term enrichment analysis to identify the BPs and pathways that are more strongly associated with the given gene list. The list of DEGs was submitted to DAVID to obtain enriched BPs and pathways.

\subsection{Kolmogorov-Smirnov test}

The set of consecutively up- and down-regulated DEGs is conjectured possibly to cluster among the top-ranked DEGs, such that up- and down-regulated DEGs tend to have smaller $p_{\text {eBayes }}$-values. The Kolmogorov-Smirnov test (KS-test) is a distribution-free test that compares the cumulative distributions of two sets of data. It was used herein to seek the difference between two distributions: (i) the consecutively upand down-regulated DEGs and (ii) the non-consecutively up- and down-regulated DEGs. The top-ranked DEGs were divided into 10 groups by using three different ways of partitions for the $K S$-test. DEGs were sorted in order of ascending $p_{\text {eBayes }}$-value. Started with a total of 7245 DEGs, we examined the following three cases of partitions: (a) using a constant ratio of 0.6 , then the grouping started from 7245 down to 73 , (b) subtraction using a constant difference of 800 , then the grouping started from 7245 down to 45 and (c) subtraction using multiple constant differences, then the grouping started from 7245 down to 125 .

\subsection{Drug repurposing - IC50 measurements}

The drug-gene interaction database, $\mathrm{cMap},{ }^{16}$ is utilized herein to find potential drugs for treating VSMC-associated diseases. CMap drugs' information (cMap name,

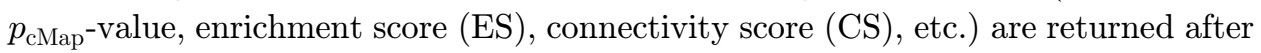
query. Both ES and CS lie between -1 and +1 .

According to the cMap webpage (http://portals.broadinstitute.org/cmap/help_topics_frames.jsp), a negative/positive ES or CS indicates that the perturbagen tended to reverse/induce the expression of the query signature. ${ }^{16,17}$ Namely, a drug with a lower negative ES or CS indicates that it is more likely to be a potential drug. 
After processing the DEGs without/with using ClusterONE, both sets of up- and down-regulated DEGs/clusters are used to query cMap. Potentially repurposed drugs with $p$-value, $p_{\text {cMap }}$, of less than 0.05 , and both ES and CS of less than 0 are identified. The results of a few drug identifications are supported by the 3-(4, 5dimethythiazol-2-yl)-2, 5-diphenyl tetrazolium bromide (MTT) assay or clonogenic assay data. The accuracy of identification of drugs are decided according to IC50 measurements.

Cell viability (the IC50 values) was determined by performing the MTT and clonogenic assay. Relevant protocols have been described in our earlier works. ${ }^{15,43}$ Two non-small cell lung cancer cell lines, A549 and H460, were used in both the MTT and clonogenic assay. For the MTT test, cells were seeded in a 96-well microplate for $16-20 \mathrm{~h}$ and treated with the indicated drugs. After drug treatment for $72 \mathrm{~h}, 50 \mu \mathrm{L}$ MTT solution $(2 \mathrm{mg} / \mathrm{mL})$ per well was added and incubated at $37^{\circ} \mathrm{C}$. Two hours later, $150 \mu \mathrm{L}$ liquid per well was removed; DMSO was added, and the absorbance at $570 \mathrm{~nm}$ was detected using an ELISA reader (Infinite ${ }^{\circledR}$ M1000, TECAN, Switzerland). The untreated groups were regarded as $100 \%$ viable.

For the clonogenic assay, cells were seeded in six-well plates at 500 cells/well for 7-10 days. Each well contained 1.5 mL RPMI medium for culturing and tested drugs were added $24 \mathrm{~h}$ after seeding with the cells. The medium and drugs were changed once on day four. Following treatment, the cells were washed with PBS, and the colonies were fixed (acetic acid: methanol, 1:3) and stained with $0.5 \%$ crystal violet in methanol. After removing the excess crystal violet and rinsing with tap water, the colonies were counted manually.

Furthermore, the "ClinicalTrials.gov results database" (https://clinicaltrials. gov/) was used to find drugs that are undergoing clinical trials.

\subsection{Identify drug-targeted DEGs, miRNAs and antimiRs}

Drug targets were obtained by submitting potential drugs to two independent databases - DrugBank ${ }^{44}$ and NCBI PubChem Compound, ${ }^{45}$ and the results were compared with both consecutively up- and down-regulated DEGs to identify drugtargeted DEGs.

In this work, two miRNA target gene resources - miRTarBase (version 6.0) ${ }^{46}$ and TarBase (version 7.0) ${ }^{47}$ — are used to identify miRNAs that hybridize drugtargeted DEGs, the miRNA-target regulatory relations are obtained, and these miRNAs may be silenced or inhibited using antimiRs. MiRTarBase has accumulated many miRNA-target interactions which are validated experimentally and collected by reporter assay, western blot, microarray and sequencing experiments. ${ }^{46}$ TarBase provides high quality experimentally validated miRNA:gene interactions, and utilized detailed meta-data to enhance the confidence of these interactions. ${ }^{47}$

The set of overlapped drug-targeted DEGs and miRNAs are shown in grey in the bottom two boxes in Fig. 2. Over-expressed miRNAs may down-regulate certain 
genes, inducing VSMC phenotypic transition. Certain drugs may target those downregulated DEGs and restore their expression levels.

As described in the introduction, antimiR-based miRNA therapeutics may be useful in inhibiting the aberrant expression of miRNA as antimiRs can be used to target over-expressed miRNAs and silence their expression, inhibiting phenotypic transitions. AntimiRs are molecules that have potential in treating VSMC-associated diseases. Accordingly, antimiRs that can hybridize the group of miRNAs that target down-regulated DEGs are identified. The silencing of the miRNAs enables the expression of down-regulated DEGs to be restored; therefore, these antimiRs have potential in developing antimiR-based miRNA therapy.

\section{Results}

\subsection{DEGs and their related functional terms}

A total of 7245 DEGs were identified by eBayes analysis; these DEGs were compared with a CVD-associated gene list that was established by assembling information from SABiosciences (QIAGEN company) ${ }^{48,49}$ and a literature review. At least 86 genes are relevant to the formation of CVDs. Among these 86 genes, 48 are associated with the proliferation of VSMCs. As indicated in Table 1, these 48 DEGs are labeled with the following functional terms; "response to stress", "apoptosis", "blood coagulation and circulation", "adhesion molecules", "extracellular molecules", "lipid transport and metabolism", "cell growth and proliferation" and "transcription regulators." 48

A website has been established to record our findings, which can be accessed at http://ppi.bioinfo.asia.edu.tw/vsmc/ by clicking the "OxLDL-induced" hyperlink. It provides (i) lists of CVD-associated genes that were compiled from SABiosciences and a literature review and (ii) the 48 VSMC proliferation-associated DEGs along with their references.

Table 1. The 48 DEGs of the VSMC-associated diseases, and their functional terms.

\begin{tabular}{|c|c|c|c|c|c|}
\hline \multicolumn{6}{|c|}{ Gene name (functional terms) } \\
\hline$A B C A 1(6)$ & $C F L A R(2)$ & $F G F 2(5,7)$ & $I L 6(1)$ & $M M P 2(5)$ & $\operatorname{SERPINE} 1(3,5)$ \\
\hline$B C L 2 A 1(2)$ & $C O X 1(1,6,7)$ & $F N 1(1,4,5)$ & ITGA2 $(3,4)$ & NFKB1 $(1,2,8)$ & $S O D 1(1)$ \\
\hline$B I R C 3(2)$ & $C S F 1(7)$ & $\operatorname{HBEGF}(5,7)$ & $I T G A 5(4)$ & $N P Y(3,5,7)$ & $T G F B 1(2,7)$ \\
\hline$C A T(2,7)$ & $C S F 2(1,5,7)$ & $I C A M 1(4)$ & $K L F 2(8)$ & PDGFA $(3,5,7)$ & TGFB2 (7) \\
\hline$C C L 2(1,2,4,5)$ & $C T G F(1,4,5,7)$ & $\operatorname{IFNAR2}(1,5)$ & $L D L R(6)$ & $\operatorname{PDGFB}(1,3,5,7)$ & $T I M P 2(7)$ \\
\hline CCL5 $(1,4,5)$ & $C X C L 1(1)$ & $I L 1 A(2,5,7)$ & $\operatorname{LIF}(5,7)$ & PLIN2 (6) & TNFAIP3 $(2,8)$ \\
\hline$C D 44(4)$ & $E G R 1(8)$ & $I L 1 B(4)$ & $M M P 1(5)$ & $P P A R A(6,8)$ & $\operatorname{VEGFA}(1,4,5,7)$ \\
\hline $\mathrm{CDH} 5$ (4) & ENG $(3,4)$ & $I L 1 R 1(1)$ & $M M P 14(5)$ & PPARG $(1,6,8)$ & $\operatorname{VWF}(1,3,4,5)$ \\
\hline
\end{tabular}

Notes: Numbers ' 1 ' to ' 8 ' denote '(1) response to stress', '(2) apoptosis', '(3) blood coagulation and circulation', '(4) adhesion molecules', '(5) extracellular molecules', '(6) lipid transport and metabolism', ' (7) cell growth and proliferation' and '(8) transcription regulators', respectively. 


\subsection{GO enrichment analysis}

DAVID can only process a limited number of input genes. Therefore, the top 3000 DEGs (with adjusted $p$-values, $p_{\text {eBayes, }}$ of less than $5.48 \mathrm{e}-03$ ) were submitted to DAVID for clustering, yielding enriched BP- and pathway-related terms.

An enrichment study of the following two sets of genes was also conducted.

(1) A total of 448 consecutively up- and 452 consecutively down-regulated DEGs that were filtered from the top 7245 DEGs, and

(2) A total of 283 consecutively up- and 278 consecutively down-regulated DEGs that were selected from the top 3000 DEGs.

Only BP or pathway terms with a $p$-value, $p_{\mathrm{GO}}$, of less than 0.05 are retained. These enriched BPs or pathways are associated with cancer- or cell regulation-related events. Our results demonstrate that many DEGs participate in the cell proliferation or apoptosis regulatory pathways, which result in the VSMC-associated diseases or cancer.

Details of the top two clusters of information about enriched BPs and pathways can be found at http://ppi.bioinfo.asia.edu.tw/vsmc/ by clicking the "OxLDLinduced" hyperlink. This website provides important genetic information. Including chromosomal locations, Unigene ID, pathways, and adjusted $p_{\text {eBayes}}$-values.

\subsection{KS test}

To perform the KS-test, 7245 DEGs $\left(p_{\text {eBayes }}<0.05\right)$ were sorted in order of ascending

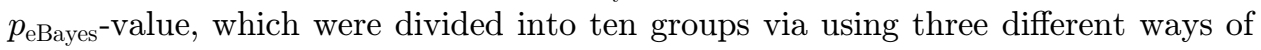
partitions (in Sec. 2.4). The maximum difference $(D)$ between the cumulative distributions of (i) the consecutively up- and down-regulated DEGs and (ii) the nonconsecutively up- and down-regulated DEGs, for the three cases were determined. The $D$ values for the three cases studies are given by: $D_{a}=0.3, D_{b}=0.3$ and $D_{c}=0.4$, with a corresponding $p$-value, $p_{K S}$, of $0.787,0.787$ and 0.418 , respectively. These results do not suggest that the consecutively up- and down-regulated DEGs tend to cluster within the top-ranked DEGs. Details of the $K S$-test results and comparative plots can be found at http://ppi.bioinfo.asia.edu.tw/vsmc/ by clicking the "OxLDL-induced" hyperlink.

\subsection{Potential repurposed drugs for VSMC-associated diseases}

Started with the 448 up- and 452 down-regulated DEGs, a total of 298 up- and 322 down-regulated DEGs were retained for analysis, because certain DEGs are not recorded by cMap. 37 drugs with both ES and CS of less than 0 were identified $\left(p_{\text {cMap }}\right.$-value $\left.<0.05\right)$. These drugs have the potential to be repurposed to inhibit the VSMC-associated diseases. Among these drugs, three (8.1\%) - GW-8510, 8-azaguanine and camptothecin — had experimentally determined IC50 activities, but 
Table 2. The list of identified potential drugs for the VSMC-associated diseases.

\begin{tabular}{|c|c|c|c|c|c|c|c|}
\hline \multirow[b]{2}{*}{ Drug name } & \multicolumn{4}{|c|}{ Potential drugs from cMap } & \multicolumn{3}{|c|}{ IC50 activities } \\
\hline & $\mathrm{ES}$ & $p$-value & Cell line & CS & & $\Gamma \mathrm{T}$ & Clonogenic \\
\hline Alsterpaullone & -0.998 & 0.00004 & PC3 & -0.928 & & & \\
\hline Spaglumic acid & -0.992 & 0.00022 & HL60 & -0.778 & & & \\
\hline Lidocaine & -0.989 & 0.00026 & PC3 & -0.895 & & & \\
\hline Camptothecin & -0.981 & 0.00082 & MCF7 & -0.764 & $\mathrm{O}$ & $<1$ & - \\
\hline$H-7$ & -0.977 & 0.00107 & PC3 & -0.719 & & & \\
\hline CP-944629 & -0.975 & 0.00129 & PC3 & -0.78 & & & \\
\hline Disopyramide & -0.968 & 0.00229 & MCF7 & -0.699 & & & \\
\hline 0179445-0000 & -0.788 & 0.0041 & MCF7 & -0.545 & & & \\
\hline Tiletamine & -0.948 & 0.00588 & MCF7 & -0.6 & & & \\
\hline Famotidine & -0.939 & 0.00767 & PC3 & -0.753 & & & \\
\hline Baclofen & -0.937 & 0.00821 & PC3 & -0.803 & & & \\
\hline Demeclocycline & -0.935 & 0.00891 & PC3 & -0.524 & & & \\
\hline Scopolamine & -0.931 & 0.00984 & MCF7 & -0.627 & & & \\
\hline Tolbutamide & -0.734 & 0.01005 & MCF7 & -0.708 & & & \\
\hline Netilmicin & -0.725 & 0.01166 & PC3 & -0.743 & & & \\
\hline Guaifenesin & -0.925 & 0.01171 & PC3 & -0.534 & & & \\
\hline Neomycin & -0.918 & 0.01352 & PC3 & -0.82 & & & \\
\hline Fluocinonide & -0.918 & 0.01354 & PC3 & -0.69 & & & \\
\hline Bromopride & -0.917 & 0.01416 & PC3 & -0.575 & & & \\
\hline Tolfenamic acid & -0.915 & 0.01453 & PC3 & -0.724 & & & \\
\hline Flumetasone & -0.914 & 0.01497 & PC3 & -0.655 & & & \\
\hline GW-8510 & -0.913 & 0.01529 & PC3 & -0.855 & $\mathrm{O}$ & $>5$ & - \\
\hline Etomidate & -0.906 & 0.01795 & MCF7 & -0.612 & & & \\
\hline Sulfamethoxazole & -0.904 & 0.01845 & PC3 & -0.688 & & & \\
\hline Flucytosine & -0.692 & 0.01936 & PC3 & -0.651 & & & \\
\hline Hydroxyachillin & -0.895 & 0.02205 & MCF7 & -0.538 & & & \\
\hline Moracizine & -0.682 & 0.0222 & MCF7 & -0.641 & & & \\
\hline Fenbufen & -0.892 & 0.02322 & PC3 & -0.678 & & & \\
\hline Edrophonium chloride & -0.892 & 0.02342 & PC3 & -0.907 & & & \\
\hline Suprofen & -0.882 & 0.02827 & MCF7 & -0.64 & & & \\
\hline Cinnarizine & -0.88 & 0.02897 & MCF7 & -0.634 & & & \\
\hline Bucladesine & -0.651 & 0.03503 & MCF7 & -0.625 & & & \\
\hline Bupropion & -0.86 & 0.03891 & MCF7 & -0.552 & & & \\
\hline 8-azaguanine & -0.86 & 0.03899 & MCF7 & -0.67 & $\mathrm{O}$ & $>5$ & null \\
\hline Prestwick-675 & -0.641 & 0.03991 & MCF7 & -0.667 & & & \\
\hline Ethotoin & -0.857 & 0.04066 & PC3 & -0.645 & & & \\
\hline Sisomicin & -0.849 & 0.04587 & MCF7 & -0.523 & & & \\
\hline
\end{tabular}

Notes: 'ES' denotes enrichment score, 'CS' denotes connective score. Italics fonts denote drugs under clinical trial. '-' denotes not available, and 'O' denotes one of the following measurements: MTT assay, clonogenic assay or both.

none is undergoing clinical trials. Table 2 presents the cMap and IC50 information for these 37 drugs. Among them, ten (27\%) are under clinical trials for CVD-associated diseases. Table 3 summarizes the clinical trial identifiers and information about the clinical studies. These 10 drugs have higher potential to be repurposed than others to inhibit the VSMC-associated diseases. 
Table 3. cMap drugs with their clinical studies information.

\begin{tabular}{lcc}
\hline Drug name & Clinical trials identifier & Related diseases \\
\hline Lidocaine & NCT01742845 & Carotid ATs \\
H-7 & NCT00570752 & ATs \\
Disopyramide & NCT00000556/NCT02294955/NCT00589303 & CVDs, HD, AF /AF/AF, HF \\
Famotidine & NCT00683111, NCT01062516/NCT00950339 & Acute CS, Acute MI/CHD \\
Scopolamine & NCT01553513/NCT02479581 & CAD/Valvular HD \\
Neomycin & NCT01097811 & Hypertension \\
Etomidate & NCT01495949/NCT01248234/NCT00415701 & Valvular HD, CAD/Hypertension/CAD \\
Bupropion & NCT02638129/NCT00689611/NCT00181818 & CVDs/MI/CVDs, MI \\
Guaifenesin & NCT00005704/NCT00680927 & CVDs, HD/AF \\
Moracizine & NCT00000556/NCT00000526/NCT00000504 & CVDs, HD, AF/CVDs, HD, MI, \\
& & CD/CVDs, HD \\
\hline
\end{tabular}

Notes: ATs, atherosclerosis; CVDs, cardiovascular diseases; HD, heart diseases; AF, atrial fibrillation; HF, heart failure; CS, coronary syndrome; MI, myocardial infarction; CHD, coronary heart disease; $\mathrm{CAD}$, coronary artery disease; $\mathrm{CD}$, coronary diseases.

\subsection{Drugs identified by clustering analysis}

The use of ClusterONE algorithm produced only one up-regulated cluster with a size of three $\left(p_{\text {ClusterONE}}\right.$-value $\left.<0.03\right)$, as well as two down-regulated clusters with sizes of six $\left(p_{\text {ClusterONE-value }}<0.002\right)$ and three $\left(p_{\text {ClusterONE-value }}<0.03\right)$. As there were so few predicted clusters, we relaxed the threshold to accept another down-regulated cluster of three $\left(p_{\text {ClusterONE}}\right.$-value $\left.<0.08\right)$.

It was found that taking the union of the up-regulated cluster and the downregulated cluster with $p_{\text {ClusterONE}}$-value $<0.03$, it yielded the highest IC50 hit ratio, i.e. $22.2 \%$ (8/36). In other words, cMap identified 36 drugs, of which eight were verified as effective by performing IC50 experiments. Combining the up-regulated cluster with the down-regulated cluster with $p_{\text {ClusterONE-value }}<0.08$ yielded the second highest hit ratio of $14.3 \%$ (6/42). Finally, combining the up-regulated cluster with the down-regulated cluster of size six yielded the lowest hit ratio of $12.5 \%(2 / 16)$.

Notably, the hit ratios for the above three combinations are at least $12.5 \%$, exceeding the value of $8.1 \%$ that is obtained without using ClusterONE. This result suggests that the use of gene clusters gives a better hit ratio. Among the predicted drugs, one drug, 8-azaguanine was identified three times and two drugs, i.e. camptothecin and GW-8510, were identified twice in the three combinations, and they were also identified without using ClusterONE. In addition, three drugs, i.e. thioguanosine, verteporfin and azacitidine, were identified twice using ClusterONE. Interesting, there are three drugs, i.e. camptothecin, mitoxantrone and vorinostat, that have a favorable IC50 in the MTT assay $(<1 u \mathrm{M})$.

\subsection{Drug-targeted DEGs identification}

A total of 37 drugs were submitted to two independent drug databases — DrugBank and NCBI PubChem Compound - to identify their targets which were used to 
Table 4. The results of identified drug-targeted DEGs.

\begin{tabular}{lcc}
\hline cMap drug & $\begin{array}{c}\text { Drug-targeted DEGs } \\
\text { from DrugBank }\end{array}$ & $\begin{array}{c}\text { Drug-targeted DEGs from } \\
\text { NCBI PubChem compound }\end{array}$ \\
\hline Cinnarizine & HRH1 (up) & HRH1 (up) \\
Flucytosine & $D N M T 1$ (down) & Not available \\
Lidocaine & $S C N 9 A$ (up) & SCN9A (up) \\
\hline
\end{tabular}

Notes: 'Up' and 'down' denote the up- and down-regulated DEGs, respectively.

match the up- and down-regulated DEGs. The DEGs HRH1 and DNMT1 are targeted by cinnarizine and flucytosine, respectively. Also, $H R H 1$ is a drug-targeted DEG that is found in both databases. One other DEG, SCN9A, which was obtained from both DrugBank and NCBI PubChem Compound, was identified as a target of lidocaine. DNMT1 is a down-regulated DEG, whereas, both $H R H 1$ and $S C N 9 A$ are up-regulated DEGs.

Histamine and its receptors, including $H R H 1$, have an important role in promoting the formation of atherosclerotic lesions by increasing vascular permeability to low-density lipoprotein (LDL), and their signaling pathways can induce the expression of downstream genes, which are involved in ATs. ${ }^{50}$ Histamine may inhibit cardiomyocyte apoptosis and increase macrophage vitality, reducing the risk of death in the course of acute myocardial infarction. ${ }^{51}$ Up-regulated DNMT1 activity accelerated the formation of foam cells, and the over-expression of DNMT1 is a significant indication of inflammation, advanced human plaques. ${ }^{52}$ In several types of the smooth muscle cell, the expression of $S C N 9 A$ may result in cell migration, and silencing $S C N 9 A$ may suppress cell migration and prevent metastasis. ${ }^{53}$ Table 4 summarizes the identified drug-targeted DEGs.

The up- and down-regulated DEG clusters that were obtained by ClusterONE analysis were also used to query cMap. Then those identified drugs were also submitted to DrugBank and NCBI PubChem Compound to identify drug targets. No drug-targeted DEG was found, mainly because the up- and down-regulated clusters include very few DEGs.

\subsection{MicroRNA-target interactions}

MiRNAs can mediate post-transcriptional gene silencing, repress gene expression. Numerous miRNAs which have been identified as novel biomarkers and potential therapeutic targets in treating VSMC-associated diseases or cancer.

The miRNA-target regulatory relations of the three drug-targeted DEGs, $H R H 1$, $D N M T 1$ and $S C N 9 A$, are obtained by searching against the miRTarBase and TarBase databases. Table 5 summarizes the related results of identified miRNAs, their target genes, expression status, the cause of disorders and references. These predicted miRNAs may target these drug-targeted DEGs, resulting in gene silencing or degradation, promoting the VSMC-associated diseases. 
Table 5. List of drug-targeted DEGs and their identified miRNAs, associated CVDs and expression status.

\begin{tabular}{|c|c|c|c|}
\hline Drug-targeted DEG & Mature miRNA & $\begin{array}{l}\text { miRNA-associated } \\
\text { diseases }\end{array}$ & $\begin{array}{c}\text { miRNA-associated } \\
\text { dysregulation }\end{array}$ \\
\hline$H R H 1$ & $\begin{array}{l}\text { miR-124-3p } \\
\text { miR-192-5p }\end{array}$ & $\begin{array}{l}\text { Stroke } \\
\text { AF }\end{array}$ & $\begin{array}{l}\text { Up-regulated }^{58} \\
\text { Up-regulated }^{59}\end{array}$ \\
\hline$D N M T 1$ & $\begin{array}{c}\text { miR-100-5p } \\
\text { miR-126-3p } \\
\text { miR-140-5p } \\
\text { miR-148a-3p, 218-5p } \\
\frac{\text { miR-148b-3p }}{\text { miR-149-5p }} \\
\frac{\text { miR-155-5p }}{\text { miR-185-5p }} \\
\text { miR-193b-3p } \\
\text { miR-26a-5p } \\
\text { miR-29b-3p } \\
\frac{\text { miR-30b-5p }}{\text { miR-342-3p }} \\
\text { miR--877-3p, } 1260 \mathrm{~b}\end{array}$ & $\begin{array}{c}\text { ATs } \\
\text { STs } \\
\text { Stroke } \\
\text { HF } \\
\frac{\mathrm{HF}}{\mathrm{CAD}, \mathrm{MI}} \\
\overline{\mathrm{AF}, \mathrm{ATs}} \\
\frac{\mathrm{CH}}{\mathrm{AF}} \\
\mathbf{M I} \\
\mathbf{H F} \\
\frac{\mathrm{CH}, \mathrm{HF}}{\frac{\mathrm{HF}}{\mathrm{CAD}}} \\
\text { Not available }\end{array}$ & $\begin{array}{c}\text { Up-regulated } \\
\text { Down-regulated }^{61} \\
\text { Do-regulated }^{62} \\
\text { Up-regulated }^{63} \\
\text { Up-regulated } \\
\text { Down-regulated }^{64} \\
\text { Down-regulated }^{65,66} \\
\text { Up-regulated }^{67,68} \\
\text { Down-regulated }^{69} \\
\text { Up-regulated }^{67} \\
\text { Up-regulated }^{70} \\
\text { Up-regulated }^{71} \\
\text { Down-regulated }^{72,73} \\
\text { Down-regulated }^{73} \\
\text { Down-regulated }^{74} \\
\text { Dot available }^{\text {Now }}\end{array}$ \\
\hline$S C N 9 A$ & miR-335-5p & & \\
\hline
\end{tabular}

Notes: AF, atrial fibrillation; ATs, atherosclerosis; HF, heart failure; CAD, coronary artery disease; MI, myocardial infarction; $\mathrm{CH}$, cardiac hypertrophy.

Based on Table 5, miR-126 may suppress the expression of a vascular cell adhesion molecule 1 (VCAM-1) and decrease vascular inflammation. ${ }^{54} \mathrm{MiR}-155$ may prevent the development and progress of ATs, and may participate in the inflammatory response and MAPK pathway by targeting $M A P 3 K 10 .{ }^{55}$ The down-regulation of miR-193b induces the expression of the urokinase-type plasminogen activator $(u P A)$, which increases the number of VSMCs, resulting in proliferative vasculopathy with intimal hyperplasia. ${ }^{56}$ OxLDL down-regulated the expression of $D N M T 3 b$ in the primary human aortic smooth muscle cells (HASMCs) by up-regulating miR-29b, and regulated $M M P-2 / M M P-9$ genes that are involved in cell migration, suggesting that miRNA-mediated regulation is crucial in ATs. ${ }^{57}$

With respect to miRNA-target interactions, miRNA-regulated DEGs can be classified into three types of modules, which are one-to-one ( $S C N 9 A)$, one-to-many (DNMT1 and HRH1), and many-to-many (not found herein). In this work, a total of three miRNA-regulated modules are identified and shown in Fig. 3.

\subsection{MicroRNA target candidates for antimiRs}

From Table 5, it was found that the drug-targeted DEG, DNMT1, is targeted by 17 miRNAs. These miRNAs are potential candidates used in developing antimiRs in miRNA inhibition for VSMC-associated diseases. Among these 17 miRNAs, eight (47\%) exhibit up-regulated expressions (boldface words in Table 5), seven (41\%) 


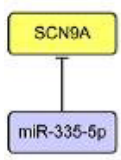

(a)

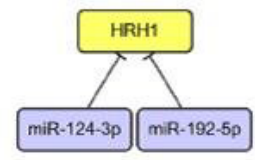

(b)

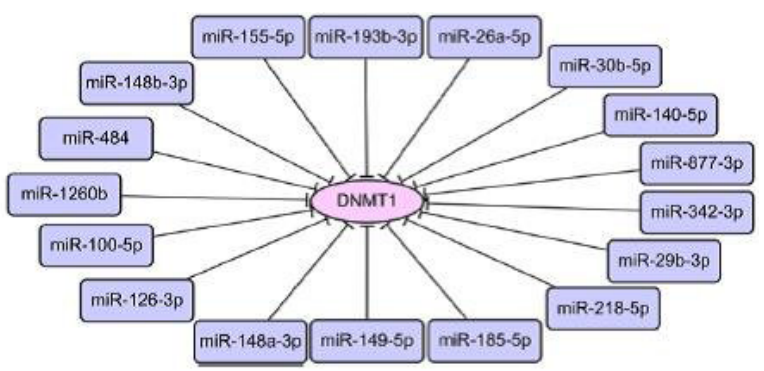

(c)

Fig. 3. The miRNA-regulated modules for the drug-targeted DEGs. (a) The one-to-one module, (b) and (c), the one-to-many module. Both $H R H 1$ and $S C N 9 A$ genes belong to the drug-targeted up-regulated DEGs; whereas DNMT1 belongs to the drug-targeted down-regulated DEG.

exhibit down-regulated expressions (underlined words in Table 5), and two (12\%) have unknown expression patterns. Based on the literature reviewing, it was found that most of the miRNAs $(88 \%)$ are expressed in the CVD-associated diseases or tissues, and suggests these predicted results are reliable herein.

In the pathogenesis of AT, the HASMCs, oxLDL induced the significant expression of miR-29b, which mediated $M M P-2 / M M P-9$ and $D N M T 3 b$ up-regulation and down-regulation, respectively. MiR-29b expression was reduced after silencing by antagomiR-29b. ${ }^{57}$ This result suggests miRNA-based therapeutic means of treating the VSMC-associated diseases by inhibiting miRNA expression. One study suggested that anti-miR-27b and anti-miR-148a can down-regulate the expression of miR-27b and miR-148a, which can feasibly be used to treat CVDs. ${ }^{75}$ The above studies provide a new perspective on the use of antimiRs as anti-disease drugs, which play an important role in research into the miRNA-based treatment of CVDs.

\section{Discussion}

Several studies have suggested that the VSMC-associated diseases and cancer have similar developmental processes. ${ }^{8-10}$ Both diseases share several molecular pathways.

In this work, processing sets of DEGs with and without ClusterONE to query cMap, the former method yielded a higher hit ratio of drugs with measured IC50 activities. Some of the identified drugs are undergoing clinical trials. Also, one miRNA-regulated DEG, DNMT1, whose miRNAs are potential targets for developing antimiR therapy, was identified. There are 37 drugs for potential repurposing and 17 putative antimiRs were identified for treating the oxLDL-induced VSMCassociated diseases. Some of the findings are supported by in vitro and clinical trial data that demonstrate the feasibility of the proposed approach.

Although important results were obtained, this work has some limitations. First, to improve the hit ratio of identification of drugs with measured IC50 activities, ClusterONE was used. Since both the up- and down-regulated clusters that were 
identified by ClusterONE are small, no drug-targeted DEG was identified. Secondly, the ClusterONE algorithm was not benchmarked against other clustering algorithms. Thirdly, although the identifications of the drugs were well supported by in vitro and clinical trial data, the cMap drugs, which were tested against cancer cells, may be toxic to VSMCs. Finally, antimiR-based therapeutics have not yet been tested in a clinical setting.

\section{Conclusions}

To examine how VSMCs react to oxLDL stimulation, a systematic study was carried out. First, in silico analysis was performed to examine how VSMCs respond to oxLDL perturbation and time-course microarray data were used to identify DEGs. Next, the identified DEGs were used to perform enrichment analysis to identify the most relevant BP and pathway terms. The cMap resource was used to identify potential drugs against the VSMC-associated diseases. Then, the DrugBank and NCBI PubChem Compound resources were utilized to identify drug-targeted DEGs for probing the course of gene therapy. Finally, the miRTarBase and TarBase resources were used to investigate the miRNA-target interactions, enabling miRNAregulated DEGs to be identified.

In conclusion, the potential of existing drugs and antimiRs for inhibition of oxLDL-induced VSMC-associated diseases was assessed, potentially supporting the repurposing of drugs and the development of alternative therapeutic strategies. Furthermore, in vitro and in vivo studies are required to explore the potential for using either existing drugs or antimiRs against the VSMC-associated diseases.

\section{Acknowledgments}

The work of Chien-Hung Huang was supported by the Ministry of Science and Technology of Taiwan (MOST) under Contract No. MOST 105-2221-E-468-016. The works of Ka-Lok Ng, Jin-Shuei Ciou, and Jeffrey J. P. Tsai were supported by MOST under Contract Nos. MOST 102-2632-E-468-001-MY3 and MOST 105-2632E-468-002. The work of Ka-Lok Ng is also supported under Contract No. MOST 1052221-E-468-016 and the Asia University grant 104-asia-03. The work of Chi-Ying F Huang is supported under Contract No. MOST 104-2627-b-010-001. The work of N Kurubanjerdjit is supported by the Mae Fah Luang University.

\section{References}

1. Huang CH, Ciou JS, Chen ST, Tzeng KR, Tsai JP Jeffrey, Kurubanjerdjit N, Ng KL, Using time-course data to identify stress- and LDL-induced microRNA target genes for vascular smooth muscle cells, Engineering Letters 23(4):307-311, 2015.

2. World Health Organization, Cardiovascular diseases - Fact sheet N317, available at http://www.who.int/mediacentre/factsheets/fs317/en/. Accessed 17 Jan 2017.

3. Rivard A, Andrés V, Vascular smooth muscle cell proliferation in the pathogenesis of atherosclerotic cardiovascular diseases, Histol Histopathol 15(2):557-571, 2000. 
4. Haudenschild CC, Pathobiology of restenosis after angioplasty, Am J Med 94(4A):40S44S, 1993.

5. Lemarié CA, Tharaux PL, Lehoux S, Extracellular matrix alterations in hypertensive vascular remodeling, J Mol Cell Cardiol 48(3):433-439, 2010.

6. Massaeli H, Hurtado C, Austria JA, Pierce GN, Oxidized low-density lipoprotein induces cytoskeletal disorganization in smooth muscle cells, Am J Physiol 277(5) Pt2:H20172025, 1999.

7. Minta J, Yun JJ, Bernard RS, Microarray analysis of ox-LDL (oxidized low-density lipoprotein)-regulated genes in human coronary artery smooth muscle cells, Cell Biol Int Rep 17(2):e00007, 2010.

8. Ross JS, Stagliano NE, Donovan MJ, Breitbart RE, Ginsburg GS, Atherosclerosis and cancer: Common molecular pathway of disease development and progression, Ann NY Acad Sci 947:271-292, 2001.

9. Ross JS, Stagliano NE, Donovan MJ, Breitbart RE, Ginsburg GS, Atherosclerosis: A cancer of the blood vessels? Am J Clin Pahtol 116(Suppl I):S97-S107, 2001.

10. Li JJ, Gao RL, Should atherosclerosis be considered a cancer of the vascular wall? Medical Hypotheses 64:694-698, 2005.

11. Jung F, Haendeler J, Goebel C, Zeiher AM, Dimmeler S, Growth factor-induced phosphoinositide 3-OH kinase/Akt phosphorylation in smooth muscle cells: Induction of cell proliferation and inhibition of cell death, Cardiovasc Res 48(1):148-157, 2000.

12. Arcaro A, Guerreiro AS, The phosphoinositide 3-kinase pathway in human cancer: Genetic alterations and therapeutic implications, Curr Genomics 8(5):271-306, 2007.

13. Jacob T, Ascher E, Alapat D, Olevskaia Y, Hingorani A, Activation of p38MAPK signaling cascade in a VSMC injury model: Role of p38MAPK inhibitors in limiting VSMC proliferation, Eur J Vasc Endovasc Surg 29(5):470-478, 2005.

14. Sebolt-Leopold JS, Herrera R, Targeting the mitogen-activated protein kinase cascade to treat cancer, Nat Rev Cancer 4(12):937-947, 2004.

15. Huang CH, Chang MH Peter, Lin YJ, Wang CH, Huang CY, Ng KL, Drug repositioning discovery for early and late stage non-small cell lung cancer, BioMed Res J Biomed Biotechnol 2014:1-13, 2014.

16. Lamb J, The connectivity map: A new tool for biomedical research, Nat Rev Cancer $7(1): 54-60,2007$.

17. Lamb J, Crawford ED, Peck D, Modell JW, Blat IC, Wrobel MJ, Lerner J, Brunet JP, Subramanian A, Ross KN, Reich M, Hieronymus H, Wei G, Armstrong SA, Haggarty SJ, Clemons PA, Wei R, Carr SA, Lander ES, Golub TR, The connectivity map: Using geneexpression signatures to connect small molecules, genes, and disease, Science 313(5795):1929-1935, 2006.

18. Ji R, Cheng Y, Yue J, Yang J, Liu X, Chen H, Dean DB, Zhang C, MicroRNA expression signature and antisense-mediated depletion reveal an essential role of MicroRNA in vascular neointimal lesion formation, Circ Res 100:1579-1588, 2007.

19. Boettger T, Beetz N, Kostin S, Schneider J, Krüger M, Hein L, Braun T, Acquisition of the contractile phenotype by murine arterial smooth muscle cells depends on the Mir143/ 145 gene cluster, J Clin Invest 119:2634-2647, 2009.

20. Liu X, Cheng Y, Zhang S, Lin Y, Yang J, Zhang C, A necessary role of miR-221 and miR-222 in vascular smooth muscle cell proliferation and neointimal hyperplasia, Circ Res 104(4):476-487, 2009.

21. Cordes KR, Sheehy NT, White MP, Berry EC, Morton SU, Muth AN, Lee TH, Miano JM, Ivey KN, Srivastava D, miR-145 and miR-143 regulate smooth muscle cell fate and plasticity, Nature 460:705-710, 2009. 
22. Stenvang J, Petri A, Lindow M, Obad S, Kauppinen S, Inhibition of microRNA function by antimiR oligonucleotides, Silence 3:1, 2012 .

23. Hennessy EJ, Moore KJ, Using microRNA as an alternative treatment for hyperlipidemia and cardiovascular disease: Cardio-miRs in the pipeline, $J$ Cardiovasc Pharmacol 62(3):247-254, 2013.

24. Marquart TJ, Allen RM, Ory DS, Baldan A, miR-33 links SREBP-2 induction to repression of sterol transporters, Proc Natl Acad Sci USA 107:12228-12232, 2010.

25. Najafi-Shoushtari SH, Kristo F, Li Y, Shioda T, Cohen DE, Gerszten RE, Naar AM, MicroRNA-33 and the SREBP host genes cooperate to control cholesterol homeostasis, Science 328:1566-1569, 2010.

26. Rayner KJ, Sheedy FJ, Esau CC, Hussain FN, Temel RE, Parathath S, van Gils JM, Rayner AJ, Chang AN, Suarez Y, Fernandez-Hernando C, Fisher EA, Moore KJ, Antagonism of miR-33 in mice promotes reverse cholesterol transport and regression of atherosclerosis, J Clin Invest 121:2921-2931, 2011.

27. Rukov JL, Shomron N, MicroRNA pharmacogenomics: Post-transcriptional regulation of drug response, Trends Mol Med 17(8):412-423, 2011.

28. Shi S, Han L, Deng L, Zhang Y, Shen H, Gong T, Zhang Z, Sun X, Dual drugs (microRNA-34a and paclitaxel)-loaded functional solid lipid nanoparticles for synergistic cancer cell suppression, J Control Release 194:228-237, 2014.

29. Barrett T, Wilhite SE, Ledoux P, Evangelista C, Kim IF, Tomashevsky M, Marshall KA, Phillippy KH, Sherman PM, Holko M, Yefanov A, Lee H, Zhang N, Robertson CL, Serova N, Davis S, Soboleva A, NCBI GEO: Archive for functional genomics data sets update, Nucleic Acids Res 41(Database issue):D991-995, 2013.

30. Irizarry RA, From CEL files to annotated lists of interesting genes, Bioinformatics and Computational Biology Solutions Using $R$ and Bioconductor, Springer-Verlag, New York, pp. 431-442, 2005.

31. Tusher VG, Tibshirani R, Chu G, Significance analysis of microarrays applied to the ionizing radiation response, Proc Natl Acad Sci USA 98(9):5116-5121, 2001.

32. Mutarelli M, Cicatiello L, Ferraro L, Grober OM, Ravo M, Facchiano AM, Angelini C, Weisz A, Time-course analysis of genome-wide gene expression data from hormone-responsive human breast cancer cells, BMC Bioinformatics 9(Suppl 2):S12, 2008.

33. Efron B, Tibshirani R, Storey JD, Tusher V, Empirical Bayes analysis of a microarray experiment, J Am Stat Assoc 96(456):1151-1160, 2001.

34. Eckel JE, Gennings C, Chinchilli VM, Burgoon LD, Zacharewski TR, Empirical Bayes gene screening tool for time course or dose-response microarray data, J Biopharm Stat 14(3):647-670, 2004.

35. Efron B, Robbins, Empirical Bayes and microarrays, Ann Stat 31(2):366-378, 2003.

36. Chen ST, Wu HF, Ng KL, A platform for querying breast and prostate cancer-related microRNA genes, Int Conf Bioinform Biomed Eng 1(1):271-274, 2012.

37. Smyth GK, Limma: Linear models for microarray data, Bioinformatics and Computational Biology Solutions Using $R$ and Bioconductor, Springer-Verlag, New York, pp. 397420, 2005.

38. Nepusz T, Yu H, Paccanaro A, Detecting overlapping protein complexes in proteinprotein interaction networks, Nat Methods 9(5):471-472, 2012.

39. Chatr-Aryamontri A, Breitkreutz BJ, Oughtred R, Boucher L, Heinicke S, Chen D, Stark C, Breitkreutz A, Kolas N, O'Donnell L, Reguly T, Nixon J, Ramage L, Winter A, Sellam A, Chang C, Hirschman J, Theesfeld C, Rust J, Livstone MS, Dolinski K, Tyers M, The BioGRID interaction database: 2015 update, Nucleic Acids Res 43(Database issue): D470-478, 2015. 
40. Huang DW, Sherman BT, Lempicki RA, Systematic and integrative analysis of large gene lists using DAVID bioinformatics resources, Nature Protocols 4(1):44-57, 2009.

41. Gene Ontology Consortium, The gene ontology (GO) project in 2006, Nucleic Acids Res 34:D322-D326, 2006.

42. Kanehisa M, Goto S, Kawashima S, Okuno Y, Hattori, The KEGG resource for deciphering the genome, Nucleic Acids Res 32(Database issue):D277-D280, 2004.

43. Lan MY, Chen CL, Lin KT, Lee SA, Yang WL, Hsu CN, Wu JC, Ho CY, Lin JC, Huang CY, From NPC therapeutic target identification to potential treatment strategy, Mol Cancer Ther 9(9):2511-2523, 2010.

44. Wishart DS, Knox C, Guo AC, Cheng D, Shrivastava S, Tzur D, Gautam B, Hassanali M, DrugBank: A knowledge base for drugs, drug actions and drug targets, Nucleic Acids Res 36(Database issue):D901-906, 2008.

45. NCBI Resource Coordinators, Database resources of the National Center for Biotechnology Information, Nucleic Acids Res 43:D6-D17, 2015.

46. Hsu SD, Tseng YT, Shrestha S, Lin YL, Khaleel A, Chou CH, Chu CF, Huang HY, Lin CM, Ho SY, Jian TY, Lin FM, Chang TH, Weng SL, Liao KW, Liao IE, Liu CC, Huang HD, miRTarBase update 2014: An information resource for experimentally validated miRNA-target interactions, Nucleic Acids Res 42:D78-D85, 2014.

47. Vlachos IS, Paraskevopoulou MD, Karagkouni D, Georgakilas G, Vergoulis T, Kanellos I, Anastasopoulos IL, Maniou S, Karathanou K, Kalfakakou D, Fevgas A, Dalamagas T, Hatzigeorgiou AG, DIANA-TarBase v7.0: Indexing more than half a million experimentally supported miRNA: mRNA interactions, Nucleic Acids Res 43(Database issue): D153-D159, 2015.

48. SABiosciences, a QIAGEN company, available at: https://www.sabiosciences.com/ rt_pcr_product/HTML/PAHS-038Z.html. Accessed 17 Jan 2017.

49. SABiosciences, a QIAGEN company, available at: https://www.sabiosciences.com/ rt_pcr_product/HTML/PAHS-174Z.html. Accessed 17 Jan 2017.

50. Rozenberg I, Sluka SH, Rohrer L, Hofmann J, Becher B, Akhmedov A, Soliz J, Mocharla P, Boren J, Johansen P, Steffel J, Watanabe T, Luscher TF, Tanner FC, Histamine H1 receptor promotes atherosclerotic lesion formation by increasing vascular permeability for low-density lipoproteins, Arterioscler Thromb Vasc Biol 30:923-930, 2010.

51. Deng L, Hong T, Lin J, Ding S, Huang Z, Chen J, Jia J, Zou Y, Wang TC, Yang X, Ge J, Histamine deficiency exacerbates myocardial injury in acute myocardial infarction through impaired macrophage infiltration and increased cardiomyocyte apoptosis, Sci Rep 5:13131, 2015.

52. Valencia-Morales MP, Zaina S, Heyn H, Carmona FJ, Varol N, Sayols S, Condom E, Ramírez-Ruz J, Gomez A, Moran S, Lund G, Rodríguez-Ríos D, López-González G, Ramírez-Nava M, de la Rocha C, A Sanchez-Flores 1, Esteller M, The DNA methylation drift of the atherosclerotic aorta increases with lesion progression, BMC Med Genomics 8:7, 2015 .

53. Meguro K, Iida H, Takano H, Morita T, Sata M, Nagai R, Nakajima T, Function and role of voltage-gated sodium channel NaV1.7 expressed in aortic smooth muscle cells, Am J Physiol Heart Circ Physiol 296(1):H211-H219, 2009.

54. Harris TA, Yamakuchi M, Ferlito M, Mendell JT, Lowenstein CJ, MicroRNA-126 regulates endothelial expression of vascular cell adhesion molecule 1, Proc Natl Acad Sci USA 105:1516-1521, 2008.

55. Zhu J, Chen T, Yang L, Li Z, Wong MM, Zheng X, Pan X, Zhang L, Yan H, Regulation of microRNA-155 in atherosclerotic inflammatory responses by targeting MAP3K10, PLoS One 7(11):e46551, 2012. 
56. Iwamoto N, Vettori S, Maurer B, Brock M, Pachera E, Jüngel A, Calcagni M, Renate E Gay, Whitfield ML, Distler JH, Gay S, Distler O, Downregulation of miR-193b in systemic sclerosis regulates the proliferative vasculopathy by urokinase-type plasminogen activator expression, Ann Rheum Dis 75(1):303-310, 2016.

57. Chen KC, Wang YS, Hu CY, Chang WC, Liao YC, Dai CY, Juo SH, OxLDL upregulates microRNA-29b, leading to epigenetic modifications of MMP-2/MMP-9 genes: A novel mechanism for cardiovascular diseases, FASEB J 25(5):1718-1728, 2011.

58. Laterza OF, Lim L, Garrett-Engele PW, Vlasakova K, Muniappa N, Tanaka WK, Johnson JM, Sina JF, Fare TL, Sistare FD, Glaab WE, Plasma microRNAs as sensitive and specific biomarkers of tissue injury, Clin Chem 55(11):1977-1983, 2009.

59. Zhao Y, Huang Y, Li W, Wang Z, Zhan S, Zhou M, Yao Y, Zeng Z, Hou Y, Chen Q, Tu X, Wang QK, Huang Z, Post-transcriptional regulation of cardiac sodium channel gene SCN5A expression and function by miR-192-5p, Biochim Biophys Acta 1852(10 PtA):2024-2034, 2015.

60. Cipollone F, Felicioni L, Sarzani R, Ucchino S, Spigonardo F, Mandolini C, Malatesta S, Bucci M, Mammarella C, Santovito D, de Lutiis F, Marchetti A, Mezzetti A, Buttitta F, A unique microRNA signature associated with plaque instability in humans, Stroke 42(9):2556-2563, 2011.

61. Zhang X, Shao S, Geng H, Yu Y, Wang C, Liu Z, Yu C, Jiang X, Deng Y, Gao L, Zhao J, Expression profiles of six circulating microRNAs critical to atherosclerosis in patients with subclinical hypothyroidism, J Clin Endocrinol Metab 99(5):E766-E774, 2014.

62. Sørensen SS, Nygaard AB, Nielsen MY, Jensen K, Christensen T, miRNA expression profiles in cerebrospinal fluid and blood of patients with acute ischemic stroke, Transl Stroke Res 5(6):711-718, 2014.

63. Kuosmanen SM, Hartikainen J, Hippeläinen M, Kokki H, Levonen A-L, Tavi P, MicroRNA profiling of pericardial fluid samples from patients with heart failure, PLoS One 10(3):e0119646, 2015.

64. Chen MC, Chang TH, Chang JP, Huang HD, Ho WC, Lin YS, Pan KL, Liu WH, Huang YK, Circulating miR-148b-3p and miR-409-3p as biomarkers for heart failure in patients with mitral regurgitation, Int J Cardiol 222:148-154, 2016.

65. Ali Sheikh MS, Xia K, Li F, Deng X, Salma U, Deng H, Wei Wei L, Yang TL, Peng J, Circulating miR-765 and miR-149: Potential noninvasive diagnostic biomarkers for geriatric coronary artery disease patients, Biomed Res Int 2015:740301, 2015.

66. van Rooij E, Sutherland LB, Thatcher JE, DiMaio JM, Naseem RH, Marshall WS, Hill JA, Olson EN, Dysregulation of microRNAs after myocardial infarction reveals a role of miR-29 in cardiac fibrosis, Proc Natl Acad Sci USA 105(35):13027-13032, 2008.

67. Wang JG, Meng X, Han J, Li Y, Luo TG, Wang J, Xin M, Xi JZ, Differential expressions of miRNAs in patients with nonvalvular atrial fibrillation, Zhonghua Yi Xue Za Zhi 92(26):1816-1819, 2012.

68. Zhang J, Zhao F, Yu X, Lu X, Zheng G, MicroRNA-155 modulates the proliferation of vascular smooth muscle cells by targeting endothelial nitric oxide synthase, Int J Mol Med 35(6):1708-1714, 2015.

69. Kim JO, Song DW, Kwon EJ, Hong SE, Song HK, Min CK, Kim do H, miR-185 plays an anti-hypertrophic role in the heart via multiple targets in the calcium-signaling pathways, PLoS One 10(3):e0122509, 2015.

70. Jaguszewski M, Osipova J, Ghadri JR, Napp LC, Widera C, Franke J, Fijalkowski M, Nowak R, Fijalkowska M, Volkmann I, Katus HA, Wollert KC, Bauersachs J, Erne $\mathrm{P}$, Lüscher TF, Thum T, Templin C, A signature of circulating microRNAs differentiates takotsubo cardiomyopathy from acute myocardial infarction, Eur Heart $J$ 35(15):999-1006, 2014. 
71. Thum T, Galuppo P, Wolf C, Fiedler J, Kneitz S, van Laake LW, Doevendans PA, Mummery CL, Borlak J, Haverich A, Gross C, Engelhardt S, Ertl G, Bauersachs J, MicroRNAs in the human heart a clue to fetal gene reprogramming in heart failure, Circulation 116(3):258-267, 2007.

72. He J, Jiang S, Li FL, Zhao XJ, Chu EF, Sun MN et al., MicroRNA-30b-5p is involved in the regulation of cardiac hypertrophy by targeting CaMKII $\delta$, J Investig Med 61(3):604-612, 2013.

73. Ellis KL, Cameron VA, Troughton RW, Frampton CM, Ellmers LJ, Richards AM, Circulating microRNAs as candidate markers to distinguish heart failure in breathless patients, Eur J Heart Fail 15(10):1138-1147, 2013.

74. Wang HW, Lo HH, Chiu YL, Chang SJ, Huang PH, Liao KH, Tasi CF, Wu CH, Tsai TN, Cheng CC, Cheng SM, Dysregulated miR-361-5p/VEGF Axis in the plasma and endothelial progenitor cells of patients with coronary artery disease, PLoS One 9(5):e98070, 2014.

75. Carlos FH, Leigh G, (WO2014201301) Anti-mir-27b and anti-mir-148a oligonucleotides as therapeutic tools for treating dyslipidemias and cardiovascular diseases, PATENT Pub. No.: WO/2014/201301, PATENT Pub. Date: 18.12.2014.

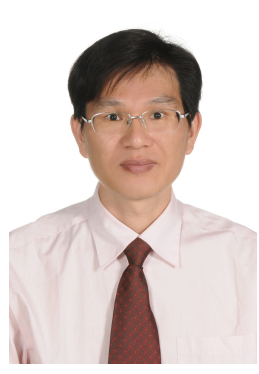

Shun-Tsung Chen is a Ph.D. student at Asia University, Taiwan. He received his M.Sc. degree in Bioinformatics from Asia University in 2010. His research fields include microarray data analysis, protein complex interaction and microRNA cancer biology.

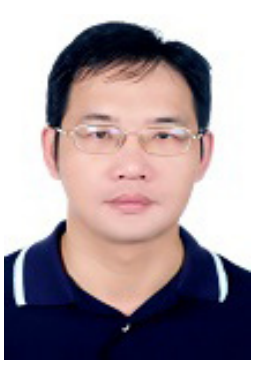

Chien-Hung Huang received his Ph.D. degree in computer and information engineering from National Tsing Hua University, Taiwan in 1999. He is a professor at the department of computer science and information engineering at the National Formosa University, Taiwan. Dr. Huang is the editorial board member of both Cancers \& Advanced Therapies and SM Journal of Biology. He has published articles in highly ranked journals such as Information Sciences, DATABASE, BMC Systems Biology, BMC Bioinformatics and Amino Acids. His research interests include graph theory, parallel computing, data hiding, biological networks, drug repositioning and microarray data analysis. 


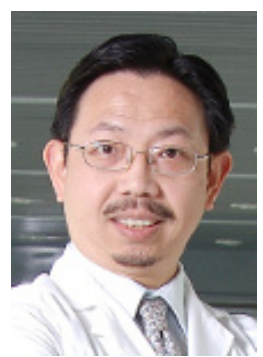

Victor C. Kok MMedSc, MD, Ph.D., FACP, is director of the cancer center and the division of medical oncology, of Kuang Tien General Hospital, Taichung, Taiwan. VCK trained as a medical oncologist at the Sun Yat-Sen Cancer Center, Taipei; certified in Medical Oncology at the European Society for Medical Oncology. He joined Asia University as an Assistant Professor. Dr. Kok actively participates as a reviewer in more than 20 SCI journals and also an editor of four oncology journals. Dr. Kok's research interests include cancer epidemiology, bioinformatics, medical oncology, internal medicine, palliative care and clinical and population health informatics.

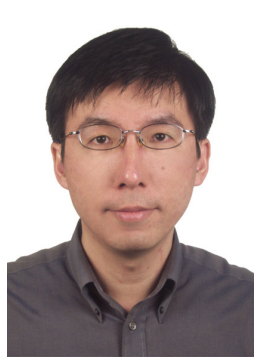

Chi-Ying F. Huang upon completion of his doctorate in Biochemistry and Biophysics at the Iowa State University in 1994, he moved to Stanford University for Postdoctoral training where he worked on the MAPK pathway. Dr. Huang joined the faculty of Division of Molecular and Genomic Medicine at the National Health Research Institutes in Taiwan in 1998, where he is an Assistant Investigator and promotes to Associate Investigator. Currently, Dr. Huang is the Professor and Chairman at the Institute of Biopharmaceutical Sciences at National Yang Ming University. He is using various function genomics techniques to identify treatment strategy for liver and lung cancer via drug repurposing.

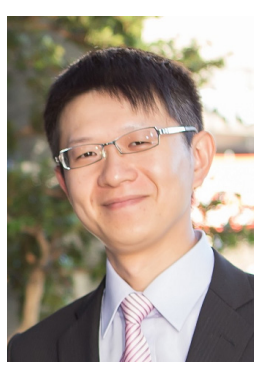

Jin-Shuei Ciou is a research assistant at Asia University, Taiwan. Currently, he commits to a novel project, precision medicine research for cancer treatments, that is based on biomedical big data analysis. He received his M.Sc. in Bioinformatics from Asia University in 2008. His research interests focus on microarray data analysis via using Bioconductor. 


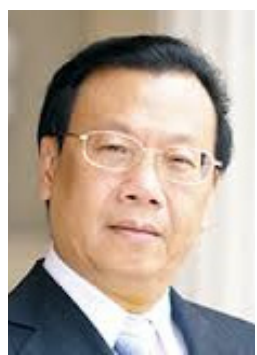

Jeffrey J. P. Tsai received his Ph.D. degree in computer science from Northwestern University, USA. He is a Chair Professor of the Department of Bioinformatics and Medical Engineering at Asia University, and also the founding and current President of Asia University. He was a Professor of the University of Illinois Chicago, a Visiting Professor at Stanford University, an Adjunct Professor at Tulane University and a Senior Research Fellow of IC2 at the University of Texas at Austin. Tsai has served on the IEEE Distinguished Speaker program, US DoD DARPA ISAT working group and on the review panel for US NSF and NIH. He received an Engineering Research Award from U.S. Engineering Foundation Society in 1988, a University Scholar Award from the University of Illinois Foundation in 1994 and an IEEE Technical Achievement Award from the IEEE Computer Society in 1997. Tsai is a Fellow of the AAAS, the IEEE and the SDPS.

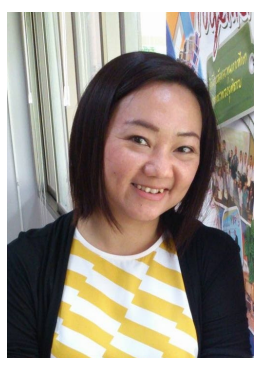

Nilubon Kurubanjerdjit received her Ph.D. in Bioinformatics from Asia University in 2014. From 2007 to 2009, she joined the faculty at the Department of Information Technology, Kasetsart University, Thailand. Currently, she is a lecturer at the School of Information Technology, Mae Fah Luang University, Thailand. Her research interest includes PPI network, cancer-related proteins and drug discovery.

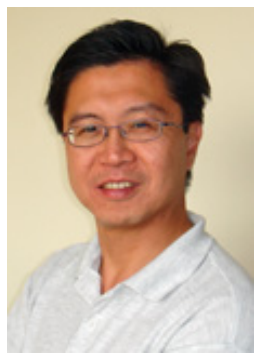

Ka-Lok $\mathrm{Ng}$ received his Ph.D. degree in physics from the Vanderbilt University at the US in 1990. He is a professor at the Department of Bioinformatics and Medical Engineering, Asia University, since February 2008. Beginning from Dec. 2009, he serves on the Editorial board of several scientific journals. Dr. Ng has published articles in highly ranked journals, in the areas of protein interactions, robustness of protein interaction networks and microRNA. 